\title{
Application of a machine learning based algorithm to online optimization of the nonlinear beam dynamics of the Argonne Advanced Photon Source
}

\author{
Louis Emery, Hairong Shang, Yipeng Sun, and Xiaobiao Huang ๑* \\ Advanced Photon Source, Argonne National Laboratory, Lemont, Illinois 60439, USA
}

(Received 29 June 2021; accepted 4 August 2021; published 18 August 2021)

\begin{abstract}
A machine learning-based optimization algorithm, the multigeneration Gaussian process optimizer, is used to optimize the nonlinear beam dynamics of the Advanced Photon Source storage ring. The dynamic aperture (DA) and the local momentum aperture (LMA) are first optimized separately with sextupole knobs. Solutions found with these optimizations are used to seed the initial population of seeds in two-objective optimizations that simultaneously optimize the DA and LMA, which lead to a distribution of solutions with different DA and LMA performances, from which a setting suitable for operation can be selected.
\end{abstract}

DOI: 10.1103/PhysRevAccelBeams.24.082802

\section{INTRODUCTION}

As storage rings push for lower emittances, sextupole magnets used in the lattices for chromaticity correction become stronger. Nonlinear beam motion due to the strong nonlinear fields in the sextupole magnets limits the region of stable motion around the design orbit. Achieving sufficient dynamic aperture (DA) and local momentum aperture (LMA) for the newer rings becomes increasingly more challenging. Optimization of storage ring nonlinear dynamics has been a critical component in the design study of a new storage ring. Advanced multiobjective optimization algorithms have been adopted in lattice design to optimize the DA and LMA [1-3].

Equally important is to achieve the design performance in DA and LMA on the real machine in spite of the inevitable differences between the lattice model and the machine, which could come from manufacturing errors, alignment errors, magnetic field measurement errors, power supply regulation errors, cross talk of magnetic fields between adjacent magnets, fringe fields, and anything else that are not precisely included in the model. Online optimization of nonlinear beam dynamics for storage rings has been proposed and demonstrated [4] in experiments, in which sextupole knobs were used to improve the DA by optimizing the injection efficiency. Since then, the method has been successfully applied to other storage rings $[5,6]$. The method has also been applied

\footnotetext{
*huangxb@anl.gov

Published by the American Physical Society under the terms of the Creative Commons Attribution 4.0 International license. Further distribution of this work must maintain attribution to the author(s) and the published article's title, journal citation, and DOI.
}

to optimize the momentum aperture (to benefit the Touschek lifetime) [7]. Online optimization of nonlinear dynamics has benefited the recent commissioning of European Synchrotron Radiation Facility - Extremely Brilliant Source [8].

In the online optimization experiments, the DA or Touschek lifetime were typically increased by $20-30 \%$ [4-7]. The successful experiments indicate that the approach is effective. It may also indicate that a large improvement in nonlinear beam dynamics could be achieved if online optimization is not previously done on a storage ring.

Optimization of DA and LMA uses the same set of sextupole knobs. However, the optimal solutions for the two objectives are typically not the same. Therefore, ideally, DA and LMA should be optimized simultaneously in a two-objective optimization problem. This has been the practice in design studies [1-3]. However, it is more difficult to do two-objective optimizations for DA and LMA in experiments because the setup for DA and LMA measurements are not compatible, the efficiency of the traditional algorithms is usually not high, and evaluation of solutions cannot be done in parallel on a real machine as is done in simulation.

At the Advanced Photon Source (APS), offline sextupole optimization was done previously with global sextupole families and local sextupole knobs around the limiting physical aperture [9]. The optimization improved both the DA and LMA simultaneously as the optimized solution effectively enlarged the physical acceptance by changing the nonlinear deformation of the phase space at the minimum aperture location. Offline and online twodimensional optimization with sextupole knobs were also done, resulting in improvement in the beam lifetime.

In this study, we conducted simultaneous optimization of DA and LMA on the APS storage ring. As far as we know, 
this is the first time such a two-objective nonlinear storage ring beam dynamics optimization is done in experiments. An efficient machine learning (ML)-based optimization algorithm, the multigeneration Gaussian process optimizer (MGGPO), was used. In the experiments, we first performed single-objective optimizations for DA and LMA, respectively. The best solutions were used to seed the initial population of solutions in the two-objective optimization. The evaluation of DA and LMA is done with two different machine and beam conditions. The approach we demonstrate here would be particularly important for the commissioning of the next generation of storage rings as their lattices have many more magnets and hence more error sources.

The paper is organized as follows: Following this introduction section, Sec. II describes the lattice setup, sextupole knobs, and the measurement of DA and LMA objectives for each lattice solution. Section III describes the optimization method and the experimental results. The conclusions are given in Sec. IV.

\section{EXPERIMENTAL SETUP}

\section{A. Lattice}

The APS is a $7-\mathrm{GeV}$ ring on a $1104 \mathrm{~m}$ circumference $[10,11]$. It consists of 40 double-bend-achromat cells, with a positive dispersion leak into the straight sections to push down the emittance. The lattice does not have full 40 -fold periodicity as the horizontal beta functions are significantly decreased at two insertion device (ID) locations and the optics in the five sectors that host the rf cavities is also slightly different. The injection efficiency is around $90 \%$ with shared horizontal disturbances between the stored beam and the injected beam. The nominal lifetime at $100 \mathrm{~mA}$ with the 24-bunch fill pattern with $1 \%$ vertical to horizontal emittance ratio (1\% coupling ratio) is about $9 \mathrm{~h}$.

The horizontal acceptance of the ring for the operation lattice is limited by a \pm 15 -mm physical aperture at the straight section ID4. This was verified with acceptance measurements done with closed orbit bumps at the location. For the acceptance measurement, an injection kicker, IK3, is fired with increasingly stronger kicks until the beam (with a short bunch train) is lost. The kicker voltage corresponding to a large fractional loss is an indication of the aperture (due to physical limitation or nonlinear beam dynamics). The measurements were repeated with the size and direction of the closed orbit bump changed. Figure 1 shows the surviving beam current as the fraction of the initial beam for four bump settings. The aperture with a -1-mm bump is the same as the nominal orbit, while the positive bump leads to an immediate aperture reduction. This indicates that the physical aperture at this location on the positive side is limiting the acceptance of the ring.

To enlarge the physical acceptance and hence allow optimization of the DA, we modified the lattice to reduce the horizontal beta function at the ID4 straight section. It

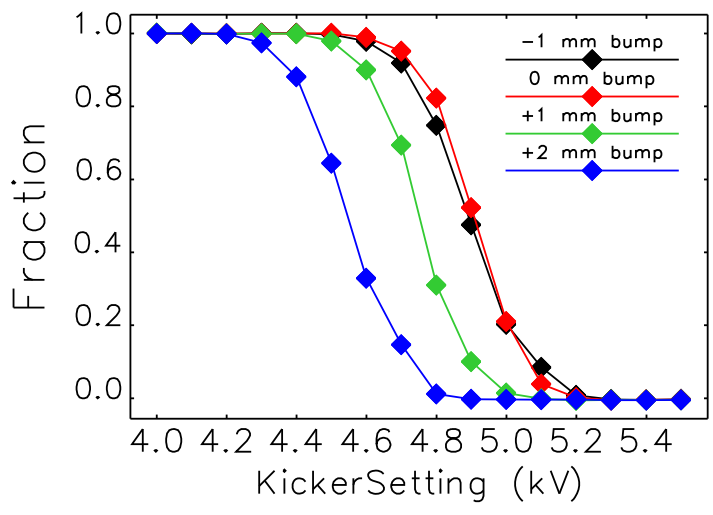

FIG. 1. Kick measurements for the horizontal aperture with various local closed orbit bump at the ID4 location. The fraction of surviving beam current vs the voltage of an injection kicker is shown.

was first reduced from $\beta_{x}=20$ to $15 \mathrm{~m}$ for one shift, and subsequently to $10 \mathrm{~m}$ on later shifts as the DA was increased through optimization.

\section{B. Tuning knobs}

An APS lattice cell has seven sextupole magnets, located symmetrically about the cell center (see Fig. 2). All 280 sextupole magnets have individual power supplies. For the low-emittance lattice, all sextupole locations have finite dispersion; therefore, changing any sextupole setpoint will change the chromaticities. To avoid changing the chromaticities by the optimization algorithm, we use combined sextupole knobs that correspond to the null space of the chromaticity response matrix, an approach adopted in Ref. [4].

In principle, we could vary each sextupole magnet independently, which leads to $280-2$ knobs that do not change the chromaticities. However, that is too large a parameter space for the optimization algorithms, and it does not necessarily help. To limit the number of knobs, sextupole magnets can be combined to form "families" according to periodicity and symmetry requirements, with magnets in the same family change by the same amount. If we require the 40 -fold periodicity and the mirror symmetry about the center of the cell, we would have only four

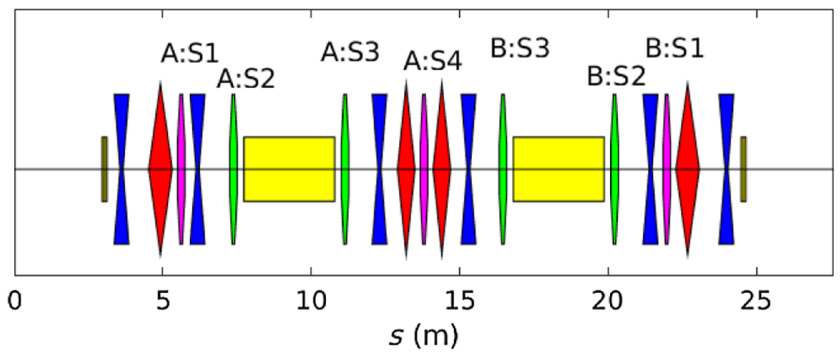

FIG. 2. A schematic of the APS lattice cell. Sextupoles are in pink or green colors and dipoles are in yellow. 
families (as sextupoles A:S1 and B:S1 will be made equal in strengths and similarly for the S2 and S3 magnets), which leaves only two independent knobs for nonlinear dynamics tuning.

In order to gain additional knobs, we allow sextupoles in the same cell to change independently. However, we maintain a 20 -fold periodicity and the mirror symmetry about the center of ID straight sections. Therefore, sextupoles $(2 N) \mathrm{B}: \mathrm{S} 1$ and $(2 N+1) \mathrm{A}: \mathrm{S} 1$ forms one family, and sextupoles $(2 N-1): \mathrm{B}: \mathrm{S} 1$ and $(2 N) \mathrm{A}: \mathrm{S} 1$ forms another family, where $N=0,1, \ldots, 19$. The $\mathrm{S} 2$ and $\mathrm{S} 3$ families are formed in similar patterns. The S4 magnets form one family. This leads to a total of seven sextupole families.

The chromaticity response matrix for the seven families is calculated with the lattice model. After singular value decomposition is done to the matrix, the five patterns with zero singular values are used as independent knobs. The tuning range for the knobs is set to allow the sextupole families change by up to $\pm 30 \%$, although that is unlikely to be achieved as it requires every knob to contribute the maximum change simultaneously.

\section{Optimization objectives}

There are two optimization objectives, DA and LMA. Since direct measurements of DA and LMA are very timeconsuming, we use surrogate quantities as the objectives in experiments. Injection efficiency is used as the DA surrogate. For injection efficiency measurement, one booster bunch with a charge of 0.5 or $1 \mathrm{nC}$ is injected into a single bucket in the ring and the current is measured for $2 \mathrm{~s}$. The efficiency is calculated from the measured current in the ring and the charge measured by a monitor located in the booster-to-storage ring transfer line. The measurement is repeated three times and the average value is used as the measured efficiency to reduce measurement noise. The measurement noise sigma is estimated to be on the $1 \%$ level. The injection kicker bump is distorted by decreasing the strengths of the last two kickers to reduce the initial injection efficiency to the $20-50 \%$ level, which leaves room for improvement and allows sensitive response to the DA changes.

Normalized beam lifetime with a high-bunch charge is used as the surrogate for the LMA. In the measurement, $24 \mathrm{~mA}$ of total beam current is stored evenly in six bunches. Lifetime is measured by monitoring the beam current for $20 \mathrm{~s}$ and fitting the data points to a linear curve with respect to time. During lifetime measurement, beam current is kept above $22 \mathrm{~mA}$. In the single-objective optimizations, the algorithm is paused whenever the beam current is below this level and is resumed after the beam current is replenished to $24 \mathrm{~mA}$. The lifetime is normalized by the beam current to eliminate the dependence of Touschek lifetime on bunch current from the objective function. As changes to sextupole knobs can change the coupling ratio, which in turn changes the Touschek lifetime, the lifetime is also normalized by the square root of the coupling ratio and a reference coupling ratio of $1 \%$ is used. The coupling ratio is measured by the $\mathrm{x}$-ray pinhole camera, which images the beam at a dipole source. The error sigma for the normalized lifetime measurement is about $2 \%$.

\section{Optimization algorithms}

The stochastic ML-based algorithm, MG-GPO [12], is used as the main optimization algorithm in this study. The robust conjugate direction search (RCDS) method [13] was also used. The MG-GPO algorithm works with a population of solutions generation by generation and generates new solutions with random operations similar to genetic algorithms [14] and particle swarm optimization [15]. The MG-GPO method gains high efficiency by modeling the data points with Gaussian process regression and using the posterior model(s) to filter out good trial solutions. It can be applied to optimization problems with single or multiple objectives. The method has been applied online to single-objective problems in Refs. $[16,17]$. This study is the first time it is applied to multiobjective optimizations online.

In our experiments, the population size is set to 15 . The initial population is generated randomly around the initial sextupole setting within a hyper cube with a side length equal to $10 \%$ of the parameter range. The cross-over operation is amplified by 20 times and the mutation by 10 times in the generation of candidate trial solutions before the posterior model is applied for filtering. These settings were based on previous experience of using the MG-GPO code on online applications $[16,17]$.

\section{OPTIMIZATION EXPERIMENTS}

\section{A. DA optimization}

We first optimized the DA with the five sextupole knobs. The first experiment was done for the lattice with $\beta_{x}=15 \mathrm{~m}$ at the ID4 location using the RCDS algorithm. The DA was improved by about $8 \%$. As the improved DA is close to the physical aperture again, in subsequent experiments, the $\beta_{x}=10 \mathrm{~m}$ lattice was used to allow further DA improvement.

The second optimization experiment was done with the MG-GPO algorithm, starting from the best solution found in the first experiment. The DA measured by the kicker method for the best RCDS solution with ID4 $\beta_{x}=15 \mathrm{~m}$ or $10 \mathrm{~m}$ are nearly the same. The kicker bump size was decreased to $55 \%$ to lower the initial injection efficiency to $32 \%$. The chromaticities were 8.0 for the horizontal plane and 5.5 for the vertical plane. Figure 3 shows the injection efficiency for all data points during the experiments. The algorithm ran for six generations and increased the injection efficiency to $68 \%$. The variations of the sextupole knobs are also shown. 

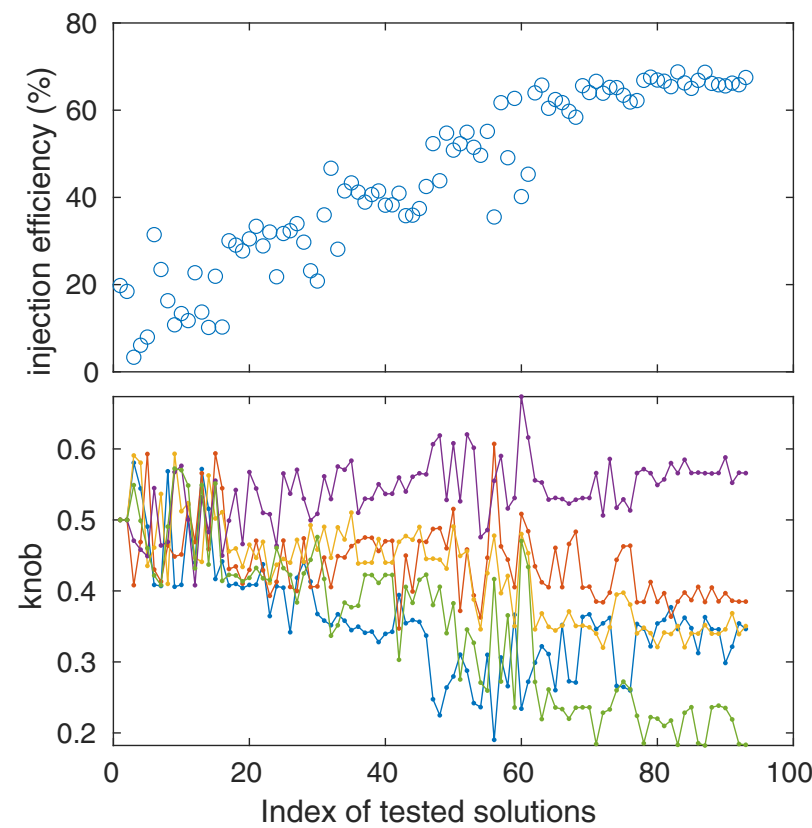

FIG. 3. Injection efficiency optimization with MG-GPO. Top: injection efficiency (the optimization objective), w/ kicker bump mismatched to make injection more challenging; bottom: sextupole knobs (normalized to the range of $[0,1]$ ).

DA measurements were done for the initial and optimized solutions and are compared. Figure 4 shows the measurement results. On top of the improvement made by the first experiment, the MG-GPO algorithm made another substantial increase in the DA. The total DA improvement is about $16 \%$. Also shown in the plot are the measured DA for two sextupole solutions found in simulation with the genetic algorithm optimization and the lattice model. Despite the predicted improvement in the DA in simulation, the measured DA for the two solutions is close to the initial sextupole setting. The DA improvement by online optimization and the fact that the optimized solution in

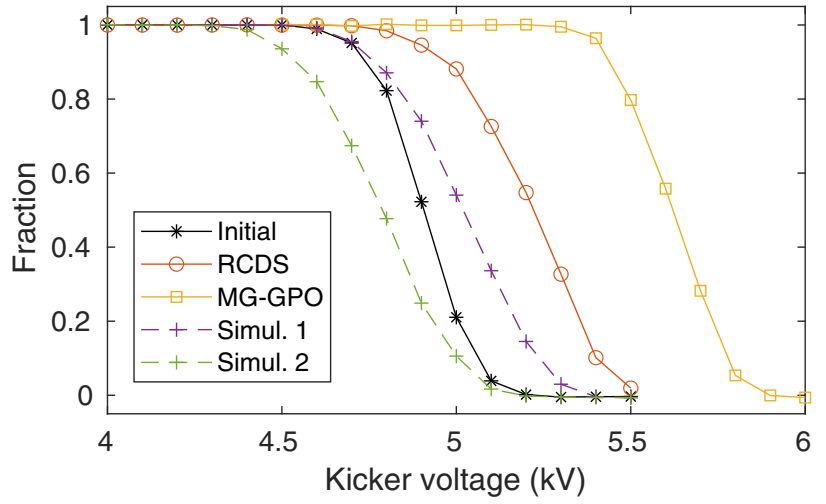

FIG. 4. Comparison of measured horizontal DA for the initial and optimized solutions. Measured DA for two solutions optimized with the lattice model are also shown. simulation does not correspond to good DA in measurement indicate that the lattice model is not accurate enough.

\section{B. LMA optimization}

The Touschek lifetime of the optimized solution from the DA optimization was measured and compared to the initial solution and was found to be about the same (269 min for the original lattice vs $265 \mathrm{~min}$ for the optimized solution). Optimization was done to improve the Touschek lifetime, starting with the lattice found in the DA optimization. The LMA was optimized with the MG-GPO algorithm by varying the same sextupole knobs to improve the normalized lifetime. Figure 5 shows the evolution of the objective function (the normalized lifetime) and the normalized sextupole knobs during the optimization run. The normalized lifetime was improved from 4.2 to $7.8 \mathrm{~h}$ in seven generations.

During the lifetime optimization, the coupling ratio changed as the sextupole setting varied. The coupling ratio values were used to compute the normalized lifetime but were not recorded in the LMA optimization. However, the range of variation can be estimated with data from the two-objective experiments (see Subsec. III C), where the coupling ratio of 75 data points varied between $1.5 \%$ and $2.1 \%$ and the average value was $1.7 \%$. The lifetime is normalized to the nominal coupling ratio of $1 \%$.

Originally, we hoped to find a good solution with a proper trade-off between DA and LMA by optimizing DA and LMA alternately. This would work if the optimized DA is partially preserved in the solution optimized for the LMA, and similarly the optimized LMA is partially

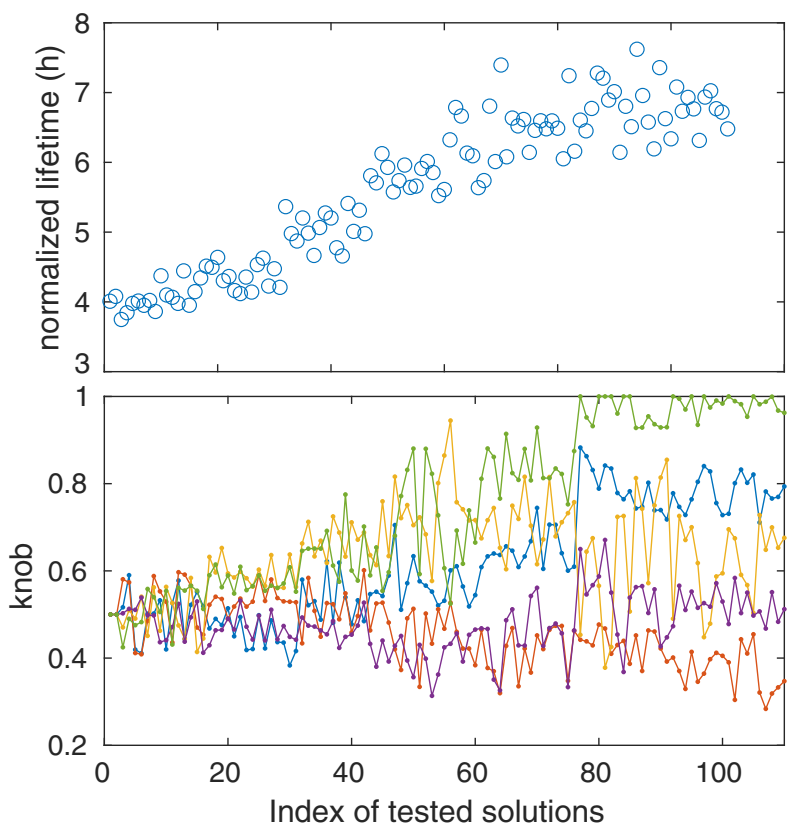

FIG. 5. The evolution of the normalized lifetime (top) and sextupole knobs (bottom) during the LMA optimization. 
preserved in the solution optimized for the DA. However, the DA of the best solution in the LMA optimization was found to be around the same level as the original sextupole setting. This indicates that an optimization that considers DA and LMA simultaneously is necessary.

\section{Two-objective optimization}

Knowing the expected best DA and LMA performances from the optimizations, a combined objective function that includes both objectives with proper normalization and weighting can be constructed and optimized. Alternatively, as we have done in this study, the two objective can be optimized simultaneously with a multiobjective optimization algorithm.

In our initial two-objective optimizations, the two objectives are evaluated one after the other for each sextupole solution. Since the measurements are done with different beam conditions, after the injection efficiency measurement is complete, the beam is dumped and refilled for the lifetime measurement. An attempt was made to reduce the total lost charge for the evaluation of each sextupole setting by reducing the number of bunches. But the lifetime measurement noise was increased. Therefore, the same six-bunch fill was used for the lifetime measurement in two-objective optimizations as for the single-objective LMA optimization. The evaluation of each solution takes about $100 \mathrm{~s}$. Experiments with this approach were done on two different shifts. The first experiment started with a population generated around two seeds, one being the best from the DA optimization, the other the best from the LMA optimization. The first experiments ran two iterations. The second experiment used the five best solutions from the first experiment as the seeds and also ran for two iterations. As this approach requires frequent changes of machine settings and frequent injections and dumps of a relatively large current of $25 \mathrm{~mA}$, the experiments were often interrupted by unexpected mishaps, such as kicker issues or tripping off of the radiation protection system.

A second approach, in which the injection efficiency and normalized lifetime are evaluated separately for all solutions in the same generation, was used later and was proven to be more efficient. As the lifetime objective is evaluated with the same $25 \mathrm{~mA}$ for all 15 solutions, the total beam loss during the experiments is significantly reduced. As less beam injection is needed, there is less chance to have interruptions due to faults. The time to complete one generation is about $18 \mathrm{~min}$, in comparison to $25 \mathrm{~min}$ for the first approach. The second approach is made possible by the generation-by-generation nature of the optimization algorithm.

The experiment with the second evaluation approach started with five solutions from the final population of solutions for the optimization with the first approach and 10 randomly generated solutions around these solutions. The optimization ran for five generations. The top plot
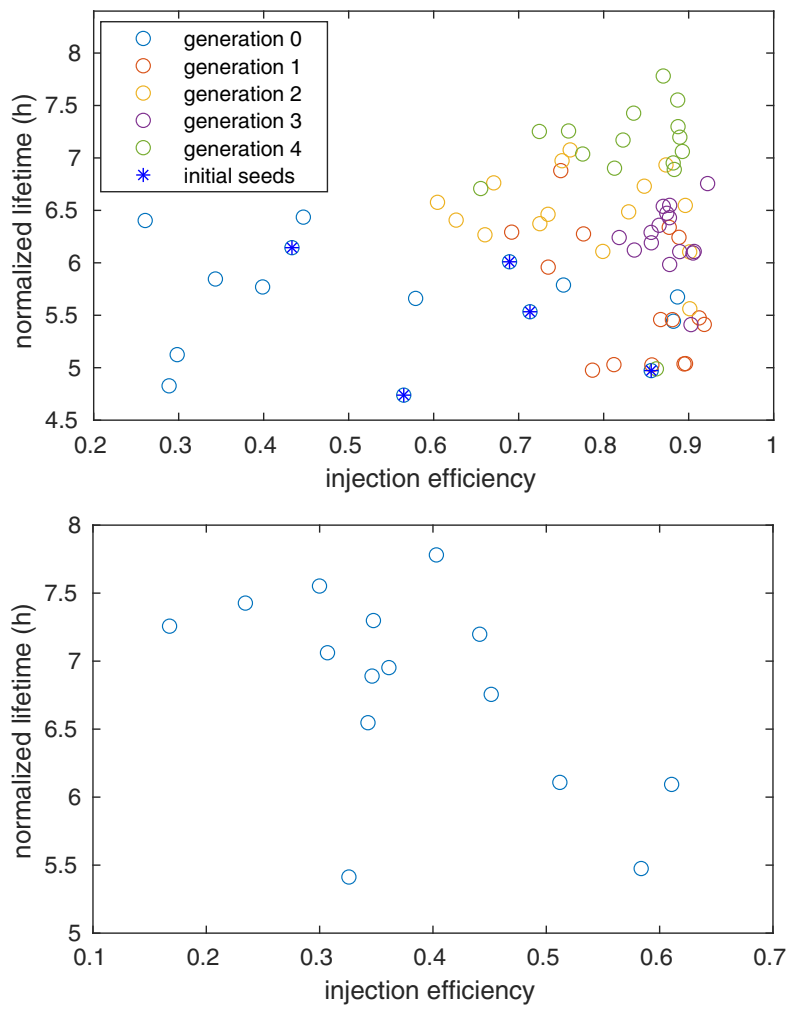

FIG. 6. The two objectives, injection efficiency and normalized lifetime, in the DA/LMA optimization. Top: evaluated solutions in the five generations, $w /$ the five initial seeds marked by '*'; the kicker bump is closed. Bottom: the best 15 solutions with injection efficiency reevaluated after changing the kicker setting to make injection more challenging.

of Fig. 6 shows the objective function values for all solutions evaluated in the generations. The injection efficiency quickly converged toward the $90 \%$ level, while the lifetime continued to improve. As the injection efficiency reaches the maximum level possible, the DA performance of the solutions is no longer well represented by the injection efficiency. For this reason, we changed the kicker setting to reduce the injection efficiency and remeasured the injection efficiency for the 15 best solutions. The results are shown in the bottom plot of Fig. 6. The distribution of the two objectives can be used to guide the selection of the sextupole setting for operation.

\section{CONCLUSION}

We conducted a series of experiments to optimize the DA and LMA for the APS storage ring with sextupole knobs, using primarily the MG-GPO method, a stochastic, MLbased optimization algorithm. A modified linear lattice with reduced horizontal beta function at the limiting physical aperture was used for the study. DA and LMA were first optimized separately with single-objective optimizations. The best solutions were used to seed the initial solution population for two-objective optimizations that 
optimize DA and LMA simultaneously. The two-objective optimization found a distribution of good solutions with different DA and LMA performances.

The two step approach for nonlinear beam dynamics optimization we demonstrated in this study can be used in the commissioning of future diffraction limited storage rings.

\section{ACKNOWLEDGMENTS}

This work was supported by the U.S. Department of Energy, Office of Science, Office of Basic Energy Sciences (BES), under Contract No. DE-AC02-06CH11357 (ANL) and BES Research and Development Project No. FWP 2020-ANL-34573.

[1] M. Borland, V. Sajaev, L. Emery, and A. Xiao, in Proceedings of the PAC 2009 (Vancouver, BC, Canada, 2009), p. 3851.

[2] L. Yang, Y. Li, W. Guo, and S. Krinsky, Phys. Rev. ST Accel. Beams 14, 054001 (2011).

[3] X. Huang and J. Safranek, Nucl. Instrum. Methods Phys. Res., Sect. A 757, 48 (2014).

[4] X. Huang and J. Safranek, Phys. Rev. ST Accel. Beams 18, 084001 (2015).

[5] D. K. Olsson, in Proceedings of IPAC2018 (Vancouver, BC, Canada, 2018), pp. 2281-2283.
[6] X. Yang, G. Ganetis, Y. Hidaka, T. Shaftan, V. Smaluk, G. Wang, L.-H. Yu, and P. Zuhoski, in Proceedings of the IPAC2019 (Melbourne, Australia, 2019).

[7] S. Liuzzo, N. Carmignani, L. Farvacque, B. Nash, T. Perron, P. Raimondi, R. Versteegen, and S. M. White, in Proceedings of the IPAC2016 (Busan, Korea, 2016), pp. 3420-3422.

[8] S. M. White et al., in Proceedings of the IPAC2021 (Brazil, 2021), p. MOXA01.

[9] M. Borland, V. Sajaev, L. Emery, and A. Xiao, Multiobjective direct optimization of dynamic acceptance and lifetime for potential upgrades of the advanced photon source, APS Technote ANL/APS/LS-319, 2010.

[10] J. Galayda, in Proceedings of the PAC 1995, Dallas, Texas, United States (1995), pp. 4-8.

[11] L. Emery, M. Borland, R. Dejus, E. Gluskin, and E. Moog, in Proceedings of the PAC 2001, Chicago, Illinois, United States (2001), pp. 2602-2604.

[12] X. Huang, M. Song, and Z. Zhang, arXiv:1907.00250.

[13] X. Huang, J. Corbett, J. Safranek, and J. Wu, Nucl. Instrum. Methods Phys. Res., Sect. A 726, 77 (2013).

[14] K. Deb, A. Pratap, S. Agarwal, and T. Meyarivan, IEEE Trans. Evol. Comput. 6, 182 (2002).

[15] J. Kennedy and R. Eberhart, in Proceedings of the ICNN'95-International Conference on Neural Networks, Perth, WA, Australia (1995), Vol. 4, pp. 1942-1948, https://doi.org/10.1109/ICNN.1995.488968.

[16] Z. Zhang, M. Song, and X. Huang, Mach. Learn. 2, 015014 (2020).

[17] H. Shang, Y. Sun, X. Huang, M. Song, and Z. Zhang, in Proceedings of the IPAC 2021, Campinas, Brazil (2021). 